\title{
CASE REPORT OF A TERM NEWBORN WITH PRENATALLY DIAGNOSED ALOBAR HOLOPROSENCEPHALY
}

\author{
Malchova E, Demova K.
}

Clinic of Neonatology, Faculty Hospital Nové Zámky, Slovak Republic

\begin{abstract}
A b s t r a c t
Holoprosencephaly is a congenital structural abnormality of brain resulting from incomplete cleavage of prosencephalon into two cerebral hemispheres. It is classified as a disorder of organogenesis and brain histogenesis and is typically associated with facial anomalies.

Holoprosencephaly is relatively rare - the incidence in live births varies between 1:10,000 - 1:15,000 but is described in 1 out of 250 spontaneously aborted embryos.

There are four major varieties of holoprosencephaly according to the degree of separation of the brain hemispheres; this division is crucial for the patient's prognosis. Three classic subtypes are alobar, semilobar, and lobar holoprosencephaly and there is also a fourth subtype called middle interhemispheric variant.

We present a case study of a term newborn with prenatally diagnosed holoprosencephaly with severe facial dysmorphism and associated health complications.
\end{abstract}

Keywords: holoprosencephaly, newborn, facial abnormalities, cleft lip and palate

\section{INTRODUCTION}

Holoprosencephaly is a structural brain malformation characterized by a failure of horizontal, transverse, and sagittal cleavage of forebrain (prosencephalon) into cerebral hemispheres. It is classified as a disorder of prosencephalic development and usually occurs between the $18^{\text {th }}$ and the $28^{\text {th }}$ day of gestation (1). As a rare abnormality, the incidence in live births varies between 1:10,000 and 1:15,000 but is much greater in aborted human embryos - 1 out of $250(1,2)$.

According to the degree of separation of the hemispheres there are four types of holoprosencephaly: alobar, semilobar, lobar holoprosencephaly, and the fourth subtype is called middle interhemispheric variant (2). The first and the most severe type is alobar form of holoprosencephaly characterized by almost complete lack of separation of the hemispheres, typically with a common midline ventricle communicating with a dorsal cyst; corpus callosum and interhemispheric fissure are completely absent. In semilobar holoprosencephaly the division of anterior hemispheres is missing but there is some degree of separation posteriorly, the fusion of deep nuclear structures is less severe, and there are posterior horns of lateral ventricles present. In the mildest lobar form, the hemispheres are almost fully separated rudimentary frontal horns may be present and parts of corpus callosum as well (genu is absent, splenium and body are present) (2). Some authors suggested a fourth subtype - middle interhemispheric variant or syntelencephaly, where only frontal and parietal regions posteriorly fail to separate (3). De Morsier syndrome (the absence of the septum pellucidum with septo - optic dysplasia) can also be considered as a mild form of holoprosencephaly. For these patients complete absence of the septum pellucidum and

Corresponding author: MUDr. Eliška Malchová; e-mail: eliska.zilkova@gmail.com

(C) 2020 Malchová Eliška

This work is licensed under the Creative Commons Attribution-NonCommercial-NoDerivs 4.0 License (https://creativecommons.org/licenses/by-nc-nd/4.0/) 
hypoplasia of the optic nerves and chiasm are typical. Visual impairment and hypothalamic - pituitary axis abnormality resulting in endocrinopathies is also common (4).

Holoprosencephaly is typically associated with many midline facial defects while the severity of facial malformations generally correlates to the severity of brain defects (1). Cyclopia (single midline fused eye), proboscis (rudimentary nasal structure), ethmocephaly (ocular hypertelorism), cebocephaly (ocular hypertelorism with a single nostril nose), or exophthalmos with median cleft lip and palate often with an absent philtrum are typically associated with alobar holoprosencephaly $(1,3)$. Less severe abnormalities are ocular hypotelorism, iris coloboma, cleft lip and palate, flattened nasal bridge, or single central incisor (5).

\section{CASE REPORT}

A 20-year-old GBS positive woman at 39 weeks of gestation came to our hospital with painful uterine contractions. The mother's history revealed that this was her fourth pregnancy (she had had one miscarriage and hade given birth spontaneously twice before - one of the kids had cleft lip and palate, the other was healthy). She was a nonsmoker and denied any chronic diseases, infections, or drug abuse during this pregnancy. The patient underwent standard screening ultrasound and examination, but no genetic testing or screening for chromosomal abnormalities. However, morphological ultrasound examination in the third trimester revealed polyhydramnios, exophthalmos, flattened midface, complete cleft lip and palate, and suspected alobar form of holoprosencephaly.

Fig. 1 Image of the newborn demonstrates a facial dysmorphism with midfacial hypoplasia, cleft lip and palate, ocular hypotelorism, exophtalmos, and single-nostil nose without nasal bones

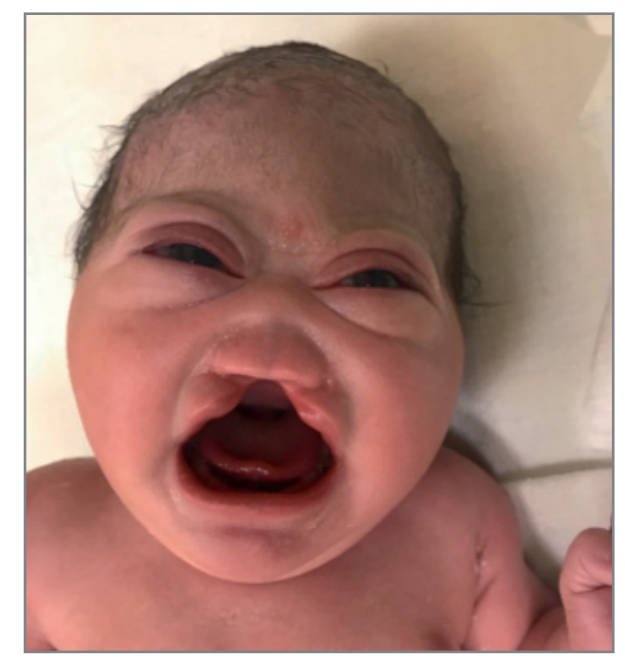

After admission the labor began with rupture of membranes with meconium stained amniotic fluid. The cardiotocography was normal, without fetal bradycardia. After $15 \mathrm{minu}$ tes a baby girl (weight $3130 \mathrm{~g}$, length $51 \mathrm{~cm}$ ) was born spontaneously without intrapartum antibiotic prophylaxis; APGAR score 9/9. Immediately after the birth there was a dominant facial dysmorphism - midfacial hypoplasia, cleft lip and palate, ocular hypotelorism with exophthalmos, single-nostril nose without nasal bones (Figure 1.)

On the second day of life the newborn developed thermal instability. To rule out possible infection we took blood culture and complete inflammatory markers with negative results, also blood culture was sterile. The child's body temperature was highly dependent on environmental conditions. The next day clinical convulsions occurred and also seizure patterns 
were detected on amplitude integrated electroencephalography (aEEG); under the phenobarbital treatment the seizures were under control. We performed a head ultrasound and also an MRI examination of the brain with findings confirming prenatal diagnosis. MRI image revealed severe congenital malformation - alobar holoprosencephaly with a single monoventricle connected to a prominent dorsal cyst and complete occipital and temporal lobes agenesis with agenesis of basal ganglia and fusion of hypoplastic frontal lobes (Figure 2. and 3.). No intracranial hemorrhage nor ischemic changes were present on the MRI image. As a part of differential diagnostics, we also performed a cardiac ECHO and renal ultrasound; both were physiological.

Fig. 2 Sagittal T2 - weighted MRI image demonstrates the most severe alobar form of holoprosencephaly with hypoplastic frontal lobes, single midline monoventricle, and complete absence of temporal and occipital lobes

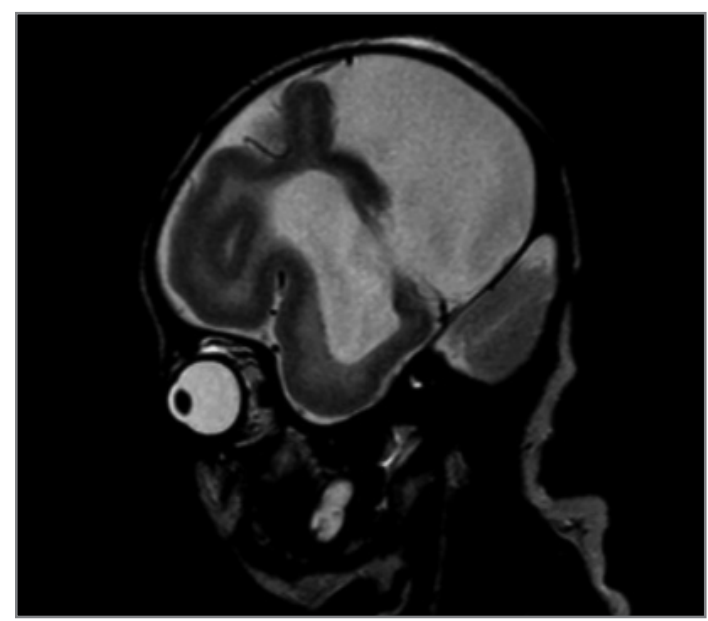

After three days of parenteral nutrition we initiated enteral feeds with special cleft palate bottle which were well tolerated by the patient and soon she started to gain weight. After 12 days of life the newborn was discharged home with the recommendation of outpatient follow - up by neurologist, geneticist, and other specialists.

Fig. 3 Axial T2 - weighted MRI image demonstrates the most severe form of holoprosencephaly with absence of interhemispheric fissure, fused hypoplastic frontal lobes, and complete agenesis of occipital lobes, temporal lobes, and basal ganglia

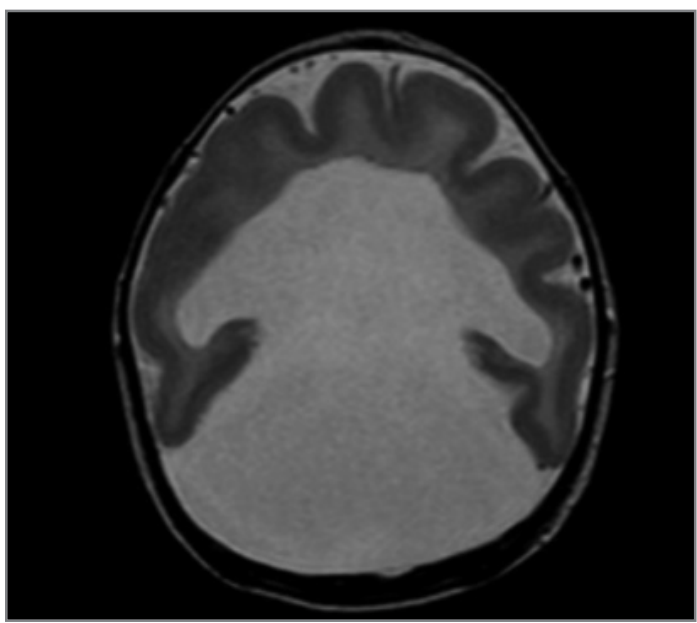




\section{DISCUSSION}

Although the etiology of holoprosencephaly is unclear, multiple genetic and environmental factors have been connected to this deformity. Most cases are sporadic, but cases of mothers with maternal diabetes, exposure to alcohol and drugs during pregnancy and also retinoic acid are mostly reported (2). Up to $45 \%$ of live births with holoprosencephaly have chromosomal abnormality, most frequently with numeric anomalies - trisomy 13 (Patau syndrome), trisomy 18 (Edwards syndrome), or triploidy (5). Some authors have suggested that holoprosencephaly can be seen in cases of malformation syndromes with normal karyotypes, typically with Smith - Lemli - Opitz syndrome (2). Also, multiple genes have been linked to holoprosencephaly - SHH (Sonic hedgehog), ZIC2 (Zinc finger protein of cerebellum 2), TGIF (transforming growth factor beta - induced factor) (6).

Prenatal diagnostics of holoprosencephaly is usually performed by ultrasonography or MRI scan. The most striking ultrasound feature is the detection of an abnormal facial profile - from mild ocular hypotelorism and microstomia to severe hypotelorism and protuberance of the nose - mouth complex resembling proboscis (7). These prenatal findings often lead to genetic counseling and testing (karyotype with microarray analysis) or termination of the pregnancy depending on the severity of the malformations. Also, the timing and mode of the delivery should be discussed before the birth of the baby (8). Although most alobar and semilobar types of holoprosencephaly can be diagnosed in the first trimester, the diagnosis of our patient was determined by a prenatal morphologic ultrasound examination at 33 weeks of gestation.

As a part of advanced diagnostics, we also included genetic testing. Since our hospital does not provide genetic testing, all of the specimens must be sent to other laboratories. During our hospital stay we were able to send the patient's blood for karyotype testing which turned out to be normal. As the child was repeatedly hospitalized shortly after her release home and placed in an institutional infant care facility due to the further health complications, other tests such as genes associated with holoprosencephaly were not performed. After our several attempts to find out the results of the testing we discovered that the child has already passed away. Consanguinity of parents was not reported.

As mentioned before, clinical findings of patients with holoprosencephaly can vary depending on the type of malformation. In this case report the patient was diagnosed with the most severe alobar form and clinical findings were midfacial hypoplasia, ocular hypotelorism, exophthalmos, cleft lip and palate, and a single nostril nose with absence of nasal bones. The connection between facial anomalies and findings on the brain has led to the phrase "the face predicts the brain". Although in our case we confirmed the most severe alobar form of holoprosencephaly, the facial anomalies were more typical for milder forms of this brain malformation.

Newborns and children with holoprosencephaly often experience several medical and neurological problems - for patients with longer survival are typical developmental delay, spasticity, dystonia, seizures, hydrocephalus, and conditions resulting from hypothalamic and brain stem dysfunction such as temperature instability, heart rate and breathing irregularities $(5,9)$. Swallowing difficulties may lead to vomiting and choking episodes which can end up as aspiration pneumonia with cardiorespiratory failure; two thirds of patients require gastrostomy tubes (2). Many of them have endocrinologic disorders - diabetes insipidus with higher serum sodium levels causes dehydration and electrolyte imbalance; hypothyroidism, hypocortisolism, and deficiency of growth hormone (2). Our patient developed secondary epilepsy with seizures, diabetes insipidus, and was also repeatedly admitted to hospital because of dyspnoe, failure to thrive, and aspiration episodes resulting in tracheostomy and percutaneous endoscopic gastrostomy.

The prognosis of patients with holoprosencephaly depends mainly on the type of disorder as well as associated anomalies but it remains poor. Many of these newborns do not survive the neonatal period and, in general, the children do not survive beyond early infancy 
(10). Only 50\% of patients with alobar holoprosencephaly survive by 6 months of age (1). Our patient died after 7 months of life due to cardiorespiratory failure.

\section{CONCLUSION}

Holoprosencephaly is a complex congenital developmental brain anomaly typically associated with facial anomalies. Multidisciplinary approach of treatment is crucial and includes many specialists because of many complications. Patients with holoprosencephaly should receive individualized treatment despite possible complications. In general, treatment should be symptomatic and supportive (11).

\section{Conflict of interests}

All authors declare that they have no conflict of interests.

\section{REFERENCES}

1. Gupta et al.: A preterm infant with semilobar holoprosencephaly and hydrocephalus: a case report. Cases Journal 2010, 3:35.

2. Swaiman, Kenneth F., Stephen Ashwal a Donna M. Ferriero.: Pediatric neurology: principles \& practice, 4th ed. Philadelphia: Mosby, 2006. ISBN 9780323033657.

3. Volpe, Joseph J.: Neurology of the newborn. 5th ed. Philadelphia: Saunders/Elsevier, 2008. ISBN 9781416039952.

4. Hahn J. S., Barnes P. D.: Neuroimaging advances in holoprosencephaly: Refining the spectrum of the midline malformation. American Journal of Medical Genetics Part C (Seminars in Medical Genetics) 2010, 154C:120-132.

5. Ionescu et al.: Alobar holoprosencephaly associated with a rare chromosomal abnormality, Case report and literature review. Medicine 2018, 97:29.

6. Firth, V.H. Overview of craniofacial clefts and holoprosencephaly. In E. TePas (Ed), UpToDate 2019. Retrieved from https://www.uptodate.com/contents/overview-of-craniofacial-clefts-andholoprosencephaly

7. El-Dessouky S.H. et al: Prenatal ultrasound findings of holoprosencephaly spectrum: Unusual associations. Prenatal Diagnosis 2020; 40:565-576.

8. Winter T. C., Kennedy A.M., Woodward J.P.: Holoprosencephaly: A Survey of the Entity, with Embryology and Fetal Imaging, RadioGraphics 2015 35:1, 275-290

9. Hahn JS, Plawner LL. Evaluation and management of children with holoprosencephaly. Pediatr Neurol 2004; 31:79-88.

10. Dubourg et al.: Holoprosencephaly. Orphanet J of Rare Diseases 2007, 2:8.

11. Amirshahi et al.: Alobar holoprosencephaly: A case report. Journal of Nursing and Midwifery Sciences 2015, 2(4): 70-74

Received: September, 7, 2020

Accepted: October, 9, 2020 